\title{
Relação entre sensibilidade plantar e controle postural em jovens e idosos
}

\section{Relationship between foot sensibility and postural control in the young and elderly}

\section{Letícia Suemi Ueda ${ }^{1}$}

Felipe Pivetta Carpes ${ }^{1,2}$

Resumo - A diminuição da sensibilidade plantar em idosos acarreta alterações na locomoção e no controle postural que podem aumentar o risco de quedas. Compreender o uso de aferências podais em idosos pode auxiliar na prevenção da perda de mobilidade e quedas. Contudo, a relação entre a sensibilidade de diferentes regiões do pé e alterações no controle postural representa uma lacuna na literatura. Com o propósito de investigar esta lacuna na literatura, o objetivo deste estudo foi avaliar a relação entre a sensibilidade plantar e o controle postural em jovens e idosos. Foram avaliados 42 participantes, organizados em dois grupos de acordo com a faixa etária (jovens ou idosos). Os participantes foram avaliados quanto às características antropométricas, sensibilidade dos pés (usando monofilamentos) e controle postural (usando plataforma de força). Os índices de sensibilidade plantar e de controle postural foram correlacionados e comparados entre os grupos. Os resultados mostraram que idosos tiveram menor sensibilidade dos pés e pior controle postural do que jovens. A amplitude ântero-posterior e a área do centro de pressão de idosos tiveram correlação com a sensibilidade plantar geral, mas não com regiões específicas do pé. Para jovens, a sensibilidade na região do antepé esteve relacionada com o melhor controle postural.

Palavras-chave: Centro de pressão; Neuromecânica; Propriocepção; Postura, Quedas.

Abstract - Impairments in the foot sensitivity in the elderly cause changes in locomotion and postural control that may increase fall risk. Understanding the use of foot afference in the elderly may help preventing loss of mobility and fall. However, there are few studies addressing the relation between the sensitivity of different foot regions and postural control. To investigating this lack in literature, the objective of our study was to assess the relationship between foot sensibility and postural control in young and elderly. Forty-two subjects volunteered to this study; they were assigned to a group according to their age (young or elderly). The participants were assessed regarding anthropometry, foot sensibility (using monofilaments) and postural control (using a force plate). The indexes of foot sensibility and postural control were correlated and compared between the groups. Elderly had worst foot sensibility and postural control than young. Center of pressure are and amplitude in antero-posterior direction were correlated with the general foot sensibility, but not with a specific point of the foot in the elderly. For young, the sensitivity in the forefoot region was related to improved postural control.

1 Universidade Federal do Pampa. Laboratório de Neuromecânica, Grupo de Pesquisa em Neuromecânica Aplicada. Uruguaiana, RS, Brasil

2 Universidade Federal de Santa Maria. Laboratório de Biomecânica. Santa Maria, RS, Brasil

Recebido em 26/06/12 Revisado em 03/10/12 Aprovado em 14/10/12

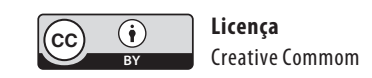




\section{INTRODUÇÃO}

Atualmente, 19 milhões de brasileiros têm 60 anos de idade ou mais. Em 2025, este número poderá atingir 32 milhões ${ }^{1}$, aumentando os custos ao sistema único de saúde. Uma estratégia para suprir boa qualidade de vida aos idosos, e consequentemente, reduzir custos à União, é compreender como os processos degenerativos associados ao envelhecimento podem ser atenuados. Neste contexto, o controle postural e quedas são temas de relevância para idosos.

A habilidade de sustentar a postura em pé envolve o processamento de informações do sistema visual, vestibular e receptores proprioceptivos, incluindo mecanorreceptores da região plantar ${ }^{2}$. O sistema somatossensorial tem como principais aferentes os mecanorreceptores em diferentes camadas da pele, os fusos musculares, órgãos tendinosos de Golgi e receptores articulares ${ }^{3}$. Os mecanorreceptores plantares informam ao sistema nervoso central sobre a interação entre a região plantar e a superfície de contato $^{4}$. Por razões como alterações nas propriedades de tecidos moles e vias de condução nervosa, o envelhecimento acarreta aumento do limiar de detecção de vibração pelos receptores cutâneos e de pressão ${ }^{5}$. Isto afeta negativamente a absorção de impactos e a captação de informações sensoriais ${ }^{6}$, dificultando a percepção de mudanças em um ambiente, como por exemplo, desníveis no solo, danos em calçamentos ou obstáculos naturais ${ }^{7}$.

As diferentes características dos tecidos moles e volumes musculares nas diferentes regiões dos pés contribuem para que a sensibilidade plantar não seja uniforme entre as diferentes partes da face plantar dos pés. Contudo, a influência de diferentes regiões do pé sobre o controle postural tem sido brevemente abordada na literatura. Patel et al. ${ }^{8}$ avaliaram a sensibilidade plantar usando monofilamentos de Semmes-Weistein para três pontos da planta do pé. Os autores discutiram que os mecanorreceptores de resposta rápida têm papel importante no controle postural, mas não foram discutidas as implicações da sensibilidade em diferentes regiões do pé. A afirmação geral foi de que a sensibilidade possui papel importante para o controle postural. Aplicando um protocolo de anestesia por hipotermia, Patel et al. ${ }^{8}$ encontraram redução na sensibilidade e capacidade de detecção tátil. Esta redução resulta da diminuição da condução elétrica, restringindo as informações dos mecanorreceptores plantares ${ }^{8}$. Stal et al. ${ }^{9}$ sugeriram que esta alteração na capacidade dos mecanorreceptores pode ser compensada por outros sistemas, fazendo com que o efeito sobre o controle postural seja de curta duração.

Dentre os estudos que discutiram aspectos relacionados com a sensibilidade de algumas regiões do pé e o controle postural ${ }^{8,9}$, nota-se a consideração de poucas regiões (apenas 3 ). Recentemente ${ }^{10}$, a relação da anatomia do pé e sua função foram discutidas, sugerindo que a complacência do pé e sua sensibilidade para pequenas deformações tem função importante para a marcha, postura em pé e resposta a perturbações. Por isso, compreender como a sensibilidade plantar contribui para o controle postural, e se alguma região do pé pode ter um papel diferenciado na discriminação 
de pequenos movimentos corporais, fornecendo informações aferentes para a regulação da postura, pode ter implicações sobre a capacidade de mobilidade e tarefas funcionais em idosos.

Baseado nestes pressupostos, nosso estudo buscou investigar a relação entre a sensibilidade plantar e o controle postural em jovens e idosos. A hipótese proposta para esta investigação foi de que, independente da idade, a sensibilidade plantar contribui para o melhor controle postural e, adicionalmente, regiões específicas do pé podem ter correlação maior com parâmetros do controle postural. Adicionalmente, a região anterior do pé, por apresentar maior mobilidade e menor tecido mole do que o calcanhar, poderia desempenhar um papel diferenciado para o controle postural.

\section{PROCEDIMENTOS METODOLÓGICOS}

\section{Participantes}

Participaram deste estudo 42 sujeitos, sedentários, com idade entre 18 e 90 anos, e organizados em dois grupos de acordo com a idade (22 jovens, sendo 7 homens, com idade entre 18 e 35 anos; 20 idosos, sendo 5 homens, com idade entre 60 e 90 anos). Todos assinaram um termo de consentimento livre e esclarecido, aprovado pelo comitê de ética em pesquisa com seres humanos da Universidade Federal do Pampa (protocolo \#08.2011). Os critérios de inclusão envolveram a capacidade de caminhar de maneira independente, sem uso de órteses ou próteses e não possuir nenhuma lesão nos membros inferiores que pudesse influenciar a locomoção ou postura, ou outras que pudessem ser resultados de neuropatias ou Diabetes (por exemplo, bolhas, úlceras ou deformações nos pés). Os critérios de exclusão envolveram histórico de doenças cerebelares, vestibulares, presença de lesões cutâneas nos pés (por exemplo, bolhas, úlceras ou deformações) e lesões graves nos membros inferiores.

Neste estudo, a relação entre sensibilidade cutânea plantar e o controle postural foi investigada em jovens e idosos. Os sujeitos foram avaliados quanto a características antropométricas, sensibilidade cutânea e controle postural em pé, com olhos fechados e descalços. Na mesma sessão, jovens foram submetidos a um protocolo de diminuição da sensibilidade cutânea. O controle postural foi monitorado durante a manutenção da postura quieta em pé, por meio de uma plataforma de força antes e depois da diminuição da sensibilidade cutânea. Os idosos não foram submetidos ao protocolo de diminuição da sensibilidade cutânea. Os resultados foram comparados entre os grupos e condições sensoriais, e a correlação entre sensibilidade plantar e o controle postural foi verificada.

\section{Avaliação da sensibilidade cutânea}

A sensibilidade cutânea na região plantar foi avaliada através de estesiometria $^{8}$, utilizando monofilamentos de nylon, de igual comprimento, com diferentes diâmetros, produzindo uma pressão padronizada sobre a pele (SORRI Bauru, Semmes-Weistein Monofilaments). A sensibilidade plantar 
foi analisada de acordo com a classificação dos monofilamentos baseada em suas cores: verde e azul: sensibilidade normal; violeta: dificuldade de discriminação de forma e temperatura; vermelho: discreta perda da sensação protetora, vulnerável a lesões; laranja: leve perda da sensação protetora; rosa: perda da sensação protetora e nenhuma resposta, perda da sensibilidade total. Para permitir a comparação entre as situações, um escore numérico foi estipulado para cada cor (verde $=1$, azul $=2$, violeta $=3$, vermelho $=$ 4 , laranja $=5$ e rosa $=6$ ). Ambos os pés foram avaliados, com a ordem do pé avaliado primeiro alternada entre os sujeitos. Em cada pé, 10 diferentes regiões foram avaliadas, conforme ilustrado na Figura 1. A sensibilidade foi determinada para cada ponto e região (ante-pé, médio-pé e retro-pé pelo somatório dos escores na região), sendo, também, como foi calculado o escore de sensibilidade para o pé inteiro (considerando o somatório de todos os pontos avaliados). As avaliações foram realizadas no mesmo ambiente, com similar condição ambiental, estando o avaliado deitado e com olhos vendados. Todos os participantes foram avaliados pelo mesmo experimentador.

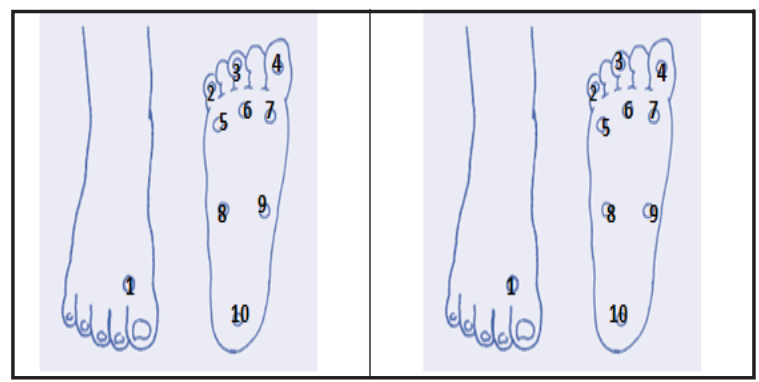

Figura 1. Ilustração dos 10 pontos para avaliação da sensibilidade plantar.

\section{Avaliação do controle postural}

O controle postural em pé foi monitorado pelas mudanças no centro de pressão $(\mathrm{CP})$ durante $30 \mathrm{~s}$, considerando a média obtida para três repetições com intervalo de $30 \mathrm{~s}$ entre elas. Os participantes foram orientados a manter os olhos fechados, com o objetivo de suprimir a informação visual para o controle postural. Para estas avaliações, o participante posicionou-se em pé sobre uma plataforma de força tridimensional (OR6 2000, AMTI Inc., EUA) instalada no nível do solo e no centro de uma sala quieta. A avaliação foi realizada (1) antes do protocolo de manipulação da sensibilidade (para jovens e idosos) e (2) depois da manipulação da sensibilidade (apenas para jovens). Os participantes permaneceram descalços e com os pés abduzidos em um ângulo de $30^{\circ}$, com os calcanhares afastados $5 \mathrm{~cm}$, braços posicionados ao lado do corpo sem executar nenhum movimento desnecessário. As informações de forças e momentos de reação do solo necessários ao cálculo do $\mathrm{CP}^{11}$ foram adquiridas a uma taxa de amostragem de $100 \mathrm{~Hz}$. Para avaliação do controle postural, foram consideradas as variáveis mais comuns relativas ao $\mathrm{CP}$, sendo elas: deslocamento ântero-posterior $\left(\mathrm{AMP}_{\mathrm{AP}}\right)$, deslocamento médio-lateral $\left(\mathrm{AMP}_{\mathrm{ML}}\right)$, área da elipse contendo $95 \%$ dos dados $\left(\mathrm{CP}_{\mathrm{AREA}}\right)$, comprimento da trajetória $\left(\mathrm{CP}_{\text {COMP }}\right)$ e velocidade resultante $\left(\mathrm{CP}_{\mathrm{VM}}\right)$. 


\section{Protocolo de anestesia para diminuição da sensibilidade}

O protocolo de anestesia foi aplicado apenas a participantes jovens, e teve o objetivo de simular a diminuição sensorial na região dos pés por meio de anestesia por hipotermia. Para a diminuição da sensibilidade cutânea na região plantar, os participantes jovens mantiveram os pés em contato com água gelada, em uma temperatura controlada entre $5^{\circ} \mathrm{C}$ e $8^{\circ} \mathrm{C}$, durante $15 \mathrm{~min}^{8}$.

\section{Análise estatística}

Os dados foram agrupados para média e desvio-padrão para cada grupo. Foi verificada a normalidade dos dados usando o teste de Shapiro-Wilk. A sensibilidade plantar dos participantes, em cada grupo, foi comparada entre o pé direito e esquerdo por meio de teste t. A partir das medidas do COP, os grupos (jovens e idosos) foram comparados entre a situação normal (jovens e idosos) e a situação anestesia por hipotermia (apenas nos jovens) por meio de análise de variância com correções de Bonferroni. A correlação entre a sensibilidade plantar e variáveis do COP foi verificada pelo teste de correlação de Pearson. Todos os testes consideraram um nível de significância de 5\%.

\section{RESULTADOS}

\section{Sensibilidade plantar x controle postural}

Apesar de não diferir entre o pé direito e esquerdo, a sensibilidade plantar foi menor no grupo de idosos comparado ao de jovens (Tabela 1); sensibilidade do ante pé $\left.\mathrm{t}_{(19)}=-6,14 ; \mathrm{p}<0,01\right]$, médio-pé $\left.\mathrm{t}_{(19)}=-6,08 ; \mathrm{p}<0,01\right]$, retro-pé $\left[\mathrm{t}_{(19)}=-7,74 ; \mathrm{p}<0,01\right]$ e sensibilidade para o pé inteiro $\left.\mathrm{t}_{(19)}=-6,11 ; \mathrm{p}<0,01\right]$.

Tabela 1. Escores de sensibilidade plantar (média e desvio-padrão) considerando as três grandes regiões do pé, e o somatório dos escores para o pé inteiro. DP, desvio-padrão.

\begin{tabular}{llcccc}
\hline & & \multicolumn{3}{c}{ Jovens } & \multicolumn{2}{c}{ Idosos } \\
\cline { 3 - 6 } & & Pé direito & Pé esquerdo & Pé direito & Pé esquerdo \\
\hline \multirow{2}{*}{ Ante-pé } & Média & 17,5 & 17,6 & $32,0^{*}$ & $32,3^{*}$ \\
& DP & 2,7 & 3,9 & 9,1 & 10,5 \\
\multirow{2}{*}{ Médio-pé } & Média & 4,5 & 4,7 & $8,8^{*}$ & $9,0^{*}$ \\
& DP & 1,2 & 1,3 & 2,7 & 3,1 \\
\multirow{2}{*}{ Retro-pé } & Média & 2,7 & 2,9 & $5,4^{*}$ & $5,4^{*}$ \\
& DP & 0,7 & 0,6 & 1,3 & 1,4 \\
\multirow{2}{*}{ Pé inteiro } & Média & 24,7 & 25,2 & $46,2^{*}$ & $46,6^{*}$ \\
& DP & 4,0 & 5,3 & 12,1 & 14,1 \\
\hline
\end{tabular}

* diferença significativa entre os grupos $(p<0,01)$; não foram observadas diferenças significativas entre os pés direito e esquerdo.

Como não houve diferença entre a sensibilidade do pé direito e esquerdo, todas as análises subsequentes foram feitas considerando apenas os dados do pé direito. Na tabela 2, são apresentados os índices de correlação entre a sensibilidade plantar geral, dada pelo somatório dos escores de todos os pontos do pé, e o controle postural para cada um dos grupos. Quando analisados os dados de todos os participantes em conjunto, apenas a $\mathrm{AMP}_{\mathrm{AP}}$ teve correlação significativa com a sensibilidade plantar geral. 
Tabela 2. Coeficientes de correlação entre a sensibilidade plantar geral e variáveis do controle postural.

\begin{tabular}{lcccccc}
\hline & & $\begin{array}{c}\mathrm{AMP}_{\mathrm{AP}} \\
(\mathrm{cm})\end{array}$ & $\begin{array}{c}\mathrm{AMP}_{\mathrm{ML}} \\
(\mathrm{cm})\end{array}$ & $\begin{array}{c}\mathrm{CP}_{\text {AREA }} \\
\left(\mathrm{cm}^{2}\right)\end{array}$ & $\begin{array}{c}\mathrm{CP}_{\text {VM }} \\
\left(\mathrm{cm}^{-1}\right)\end{array}$ & $\begin{array}{c}\mathrm{CP}_{\text {CoMP }} \\
(\mathrm{cm})\end{array}$ \\
\hline \multirow{2}{*}{ Todos os sujeitos } & $\mathrm{r}$ & $0,503^{*}$ & $-0,089$ & 0,273 & 0,152 & 0,152 \\
& $\mathrm{p}$ & 0,001 & 0,576 & 0,080 & 0,335 & 0,335 \\
\multirow{2}{*}{ Jovens } & $\mathrm{r}$ & $-0,06$ & $-0,05$ & $-0,09$ & $-0,02$ & $-0,02$ \\
& $\mathrm{p}$ & 0,78 & 0,82 & 0,68 & 0,93 & 0,93 \\
\multirow{2}{*}{ Idosos } & $\mathrm{r}$ & $0,42^{*}$ & 0,31 & $0,50^{*}$ & 0,04 & 0,04 \\
& $\mathrm{p}$ & 0,04 & 0,15 & 0,01 & 0,84 & 0,84 \\
\hline
\end{tabular}

* correlação significativa $(p<0,05)$.

Avaliando os grupos separadamente (Tabela 2), apenas a $\mathrm{AMP}_{\mathrm{AP}} \mathrm{e}$ $\mathrm{CP}_{\text {ÁREA }}$ de idosos apresentou correlação significativa com a sensibilidade para o pé inteiro. Em jovens, a sensibilidade para o pé inteiro, não teve correlação com as variáveis do CP analisadas.

Na tabela 3, são apresentados os índices de correlação entre a sensibilidade para cada ponto avaliado no pé e as variáveis do CP. Dentre jovens, os pontos que apresentaram correlação com variáveis do $\mathrm{CP}$ foram os de número $1,3,4,5$, que correspondem, respectivamente, a face dorsal do pé, superfície plantar da falange distal do terceiro pododáctilo, falange distal do hálux e parte distal do metatarso do quinto pododáctilo.

Tabela 3. Coeficiente de correlação (valor de r) entre a sensibilidade em diferentes regiões do pé e variáveis do CP para o grupo de jovens e idosos.

\begin{tabular}{|c|c|c|c|c|c|c|}
\hline Grupo & Regiões do pé & $\mathrm{AMP}_{\mathrm{AP}}$ & $\mathrm{AMP}_{\mathrm{ML}}$ & $\mathrm{CP}_{\text {AREA }}$ & $\mathrm{CP}_{\mathrm{VM}}$ & $\mathrm{CP}_{\text {СOMP }}$ \\
\hline \multirow{10}{*}{ Jovens } & Ponto 1 & $-0,03$ & $-0,20$ & $-0,05$ & $-0,43^{*}$ & $-0,43^{*}$ \\
\hline & Ponto 2 & $-0,30$ & 0,40 & 0,07 & $-0,14$ & $-0,14$ \\
\hline & Ponto 3 & $0,55^{*}$ & 0,03 & $0,48^{*}$ & 0,20 & 0,20 \\
\hline & Ponto 4 & 0,21 & 0,17 & $0,47^{*}$ & $-0,16$ & $-0,16$ \\
\hline & Ponto 5 & 0,37 & $0,48^{*}$ & $0,50^{*}$ & 0,10 & 0,10 \\
\hline & Ponto 6 & 0,11 & 0,30 & 0,23 & 0,17 & 0,17 \\
\hline & Ponto 7 & 0,40 & 0,18 & 0,36 & $-0,04$ & $-0,04$ \\
\hline & Ponto 8 & 0,41 & 0,04 & 0,23 & 0,19 & 0,19 \\
\hline & Ponto 9 & 0,20 & 0,31 & 0,22 & 0,26 & 0,26 \\
\hline & Ponto 10 & 0,40 & 0,18 & 0,37 & 0,15 & 0,15 \\
\hline \multirow{10}{*}{ Idosos } & Ponto 1 & 0,04 & $-0,02$ & $-0,04$ & 0,07 & 0,07 \\
\hline & Ponto 2 & 0,18 & 0,06 & 0,09 & 0,02 & 0,02 \\
\hline & Ponto 3 & 0,08 & $-0,08$ & $-0,02$ & 0,04 & 0,04 \\
\hline & Ponto 4 & $-0,12$ & $-0,05$ & $-0,13$ & $-0,16$ & $-0,16$ \\
\hline & Ponto 5 & $-0,14$ & $-0,32$ & $-0,30$ & $-0,20$ & $-0,20$ \\
\hline & Ponto 6 & $-0,20$ & $-0,33$ & $-0,30$ & $-0,24$ & $-0,24$ \\
\hline & Ponto 7 & $-0,29$ & $-0,33$ & $-0,36$ & $-0,21$ & $-0,21$ \\
\hline & Ponto 8 & $-0,01$ & $-0,02$ & $-0,04$ & $-0,11$ & $-0,11$ \\
\hline & Ponto 9 & $-0,13$ & $-0,07$ & $-0,15$ & $-0,19$ & $-0,19$ \\
\hline & Ponto 10 & 0,27 & 0,25 & 0,25 & 0,01 & 0,01 \\
\hline
\end{tabular}

* correlação significativa $(p<0,05)$.

Embora a Tabela 2 sugira que $\mathrm{AMP}_{\mathrm{AP}}$ e $\mathrm{CP}_{\text {AREA }}$ possam ter correlação com a sensibilidade para o pé inteiro, quando os resultados dos idosos fo- 
ram analisados, considerando cada ponto do pé em específico, não foram encontradas correlações significantes.

\section{Condição sensorial $x$ controle postural}

Os resultados da relação entre sensibilidade plantar e controle postural foram avaliadas em jovens na condição normal, jovens após anestesia e idosos (Tabela 4). Foi observado efeito de grupo para a amplitude de deslocamento ântero-posterior do $\mathrm{CP}\left(\mathrm{AMP}_{\mathrm{AP}}\right)\left[\mathrm{F}_{(2)}=11,906 ; \mathrm{p}<0,01\right]$ e para a área do $\mathrm{CP}\left(\mathrm{CP}_{\hat{A} R E A}\right)$ $\left[\mathrm{F}_{(2)}=5,229 ; \mathrm{p}<0,01\right]$. Nesse sentido, o desempenho postural foi pior em idosos. Considerando a relação com a sensibilidade plantar geral (Tabela 2), $\mathrm{AMP}_{\mathrm{AP}}$ e $\mathrm{CP}_{\text {AREA }}$ apresentaram correlação significativa com o controle postural em idosos. Não foi encontrado efeito de grupo para a amplitude de deslocamento do $\mathrm{CP}$ médio-lateral $\left(\mathrm{AMP}_{\mathrm{ML}}\right)\left[\mathrm{F}_{(2)}=1,411 ; \mathrm{p}=0,251\right]$, velocidade resultante do $\mathrm{COP}\left(\mathrm{CP}_{\mathrm{VM}}\right)\left[\mathrm{F}_{(2)}=2,660 ; \mathrm{p}=0,078\right]$ e comprimento de trajetória do $\mathrm{CP}\left(\mathrm{CP}_{\mathrm{COMP}}\right)$ $\left[\mathrm{F}_{(2)}=2,661 ; \mathrm{p}=0,078\right]$ apresentados na Tabela 4 . Os dados de $\mathrm{AMP}_{\mathrm{AP}}$ foram similares entre jovens na condição normal e anestesia $\left.\mathrm{t}_{(21)}=-1,604 ; \mathrm{p}=0,124\right]$. Contudo, ambas as condições normal $\left[\mathrm{t}_{(21)}=-5,335 ; \mathrm{p}<0,001\right]$ e anestesia em jovens $\left.\mathrm{t}_{(21)}=-3.336 ; \mathrm{p}<0,001\right]$ diferiram significativamente de idosos. Por outro lado, $\mathrm{AMP}_{\mathrm{ML}}$ foi similar entre jovens na condição normal e de anestesia $\left[\mathrm{t}_{(21)}=-1,224\right.$; $\mathrm{p}=0,235]$, assim como entre idosos e jovens na condição normal $\mathrm{t}_{(21)}=0,738$; $\mathrm{p}=0,469]$ e idosos e jovens na condição de anestesia $\left[\mathrm{t}_{(21)}=1,692 ; \mathrm{p}=0,105\right]$.

$\mathrm{O} \mathrm{CP}_{\text {AREA }}$ foi similar entre jovens na condição normal e de anestesia $\left[\mathrm{t}_{(21)}=-1,398 ; \mathrm{p}=0,177\right]$, mas idosos diferiram significativamente de jovens na condição normal $\left[\mathrm{t}_{(21)}=-3,301 ; \mathrm{p}=0,003\right]$ e dos jovens na condição anestesia $\left[\mathrm{t}_{(21)}=0,016 \mathrm{p}<0,01\right]$. Para o $\mathrm{CP}_{\mathrm{VM}}$, jovens na condição normal não diferiam da condição anestesia $\left[\mathrm{t}_{(21)}=-0,818 ; \mathrm{p}=0,422\right]$ nem de idosos $\left[\mathrm{t}_{(21)}=-1,875 ; \mathrm{p}=0,075\right]$, jovens na condição anestesia e idosos também apresentaram desempenho similar, suportando a perda de sensibilidade devido a anestesia $\left[\mathrm{t}_{(21)}=-1,585\right.$; $\mathrm{p}=0,128]$. Para $\mathrm{CP}_{\mathrm{COMP}}$ jovens na condição normal não diferiam da anestesia $\left[\mathrm{t}_{(21)}=-0,820 ; \mathrm{p}=0,422\right]$ nem dos idosos $\left[\mathrm{t}_{(21)}=-1,873 ; \mathrm{p}=0,075\right]$. Anestesia e idosos também apresentaram desempenho similar $\left[\mathrm{t}_{(21)}=-1,580 ; \mathrm{p}=0,128\right]$.

Tabela 4. Dados de amplitude de deslocamento do $\left(P\right.$ ântero-posterior $\left(A M P{ }_{A P}\right)$ e $\left(P\right.$ médio-lateral $\left(A M P P_{M L}\right)$, área do $C P\left(C P_{A ́ R E A}\right)$, velocidade do $C P\left(C P_{V M}\right)$, comprimento da trajetória do $C P(C P$ COMP $)$. Valores de média e desvio-padrão (DP) para jovens e idosos na condição normal e em jovens após anestesia. DP, desvio-padrão.

\begin{tabular}{llccc}
\hline $\mathrm{CP}$ & & Jovens & Anestesia & Idosos \\
\hline $\mathrm{AMP}_{\text {AP }}$ & Média & 1,40 & 1,74 & $3,00^{* \#}$ \\
$(\mathrm{~cm})$ & $\mathrm{DP}$ & 0,70 & 1,39 & 1,30 \\
$\mathrm{AMP}_{\mathrm{ML}}$ & Média & 2,29 & 2,56 & 2,11 \\
$(\mathrm{~cm})$ & $\mathrm{DP}$ & 0,79 & 0,73 & 1,18 \\
$\mathrm{CP}_{\text {AREA }}$ & Média & 2,22 & 2,80 & $5,18^{* \#}$ \\
$\left(\mathrm{~cm}^{2}\right)$ & $\mathrm{DP}$ & 1,75 & 2,53 & 4,87 \\
$\mathrm{CP}_{\mathrm{VM}}$ & Média & 1,86 & 1,90 & 2,07 \\
$(\mathrm{~cm} . \mathrm{s}-1)$ & $\mathrm{DP}$ & 0,24 & 0,21 & 0,47 \\
$\mathrm{CP}_{\text {COMP }}$ & Média & 55,77 & 56,94 & 61,97 \\
$(\mathrm{~cm})$ & $\mathrm{DP}$ & 7,26 & 6,26 & 14,00 \\
\hline
\end{tabular}

* diferente dos jovens na condição normal $(P<0,05)$; ${ }^{\#}$ diferente dos jovens na condição anestesia $(P<0,05) ; \%$ diferente dos idosos $(\mathrm{P}<0,05)$. 


\section{DISCUSSÃO}

Neste estudo, testamos a hipótese de que a sensibilidade na região plantar contribui para o controle postural, e que regiões específicas do pé podem ter maior correlação com parâmetros do controle postural, o que dependeria da maior modulação da ativação muscular observada, por exemplo, para a região anterior do pé, além de características mecânicas ${ }^{10}$. Dessa forma, lesões ou déficits em regiões específicas do pé que alterem a sensibilidade do pé poderiam aumentar o risco de quedas em idosos. O principal achado do presente estudo foi que a sensibilidade plantar geral em idosos teve correlação com o CP na direção ântero-posterior e área do CP, mas esta correlação não ocorreu para uma região específica do pé. Por outro lado, jovens apresentaram correlação da sensibilidade em pontos da região anterior do pé com variáveis do $\mathrm{CP}$, mas não com a sensibilidade geral do pé. Sendo assim, é difícil determinar qual região do pé possui maior relação com o controle postural em idosos.

A sensibilidade plantar foi menor em idosos. Este resultado era esperado, pois o envelhecimento acarreta alterações no sistema nervoso central e periférico, como perda de fibras mielinizadas e não mielinizadas, além da diminuição da velocidade de condução nervosa, que reduzem a capacidade de discriminação sensorial ${ }^{12}$. Adicionalmente, a diminuição do calibre das fibras nervosas e da mielinização aumenta o limiar de sensibilidade de tato leve nas mãos e pés ${ }^{12}$. Sugere que não existe diferença na discriminação de estímulos na região plantar entre os pés direito e esquerdo, independente da idade. Um fator adicional contribuindo para as perdas de sensibilidade plantar em idosos é a maior rigidez dos tecidos moles que se tornam menos perceptíveis à compressão ${ }^{13}$. Idosos oscilam mais na direção ântero-posterior do que jovens ${ }^{14}$. Contudo, como demonstrado aqui, essa diferença não se repete para a $\mathrm{AMP}_{\mathrm{ML}}$. Adicionalmente, a maior área do $\mathrm{CP}$ reflete o aumento na oscilação corporal na posição quieta em pé, frequente em idosos ${ }^{15}$.

Idosos apresentaram menor sensibilidade plantar que jovens, e mesmo com a anestesia dos pés, os jovens apresentaram controle postural melhor do que idosos. Como demonstrado pelos resultados, em jovens a sensibilidade geral do pé não teve correlação significativa com variáveis do controle postural, o que corrobora o fato de reduzida sensação tátil ter efeito negativo no controle postural apenas para sujeitos que possuem perdas sensoriais nos pés ${ }^{16}$. Dessa forma, a correlação não significativa entre a sensibilidade do pé inteiro e CP em jovens ajuda a explicar porque, na condição de anestesia, eles não tiveram o controle postural prejudicado. É bastante razoável afirmar que outros mecanismos sensoriais têm participação maior do que a informação sensorial da planta do pé no controle postural de jovens $\mathrm{s}^{8,10}$. Este resultado poderia ser explicado pelo uso de uma estratégia postural alterada, usando o tornozelo para tentar substituir a deficiência proprioceptiva da planta do pé devido à anestesia. Porém, em idosos, esse tipo de modificação na estratégia de controle postural parece não ser suficiente para contrapor os déficits sensoriais ${ }^{17}$. 
Os resultados sugerem que a região do antepé pode ter uma participação diferenciada para o controle postural em jovens, mas não para idosos; ou o envelhecimento faz com que essa característica seja perdida, o que precisa ser melhor investigado. Enquanto jovens têm a sensibilidade de uma determinada região da planta do pé correlacionada mais do que outra, com variáveis do controle postural, idosos que apresentam perdas na sensibilidade não conseguem utilizar as diferentes regiões do pé de maneira diferenciada. Este resultado suporta uma maior modulação da postura, por meio da influência da sensibilidade plantar sobre a atividade de flexores plantares e consequente participação do ante-pé18 ${ }^{18} \mathrm{~m}$ jovens, o que pode ser substituído por movimentos do joelho e/ou tronco, quando as informações sensórias da perna e pé falham, para o caso dos idosos ${ }^{19}$. Dessa forma, a redução nas redundâncias motoras para o controle postural devido à limitação no uso de informações sensoriais dos pés, ajuda a explicar o pior controle postural dos idosos. Isto é reforçado pelo fato de que estímulos leves, como os promovidos pelo toque dos dedos da mão em um apoio, serem associados com redução da pressão na região do calcanhar e ante-pé ${ }^{20}$. Sob um ponto de vista clínico, esses dados sugerem que avaliações sensoriais dos pés de idosos, visando relações com controle postural, não podem considerar apenas um ponto do pé, mas sim uma medida representativa do pé inteiro, o que vai de encontro aos resultados de estudos que avaliaram apenas pontos específicos dos pés.

As limitações do estudo envolvem não termos monitorado o COP para cada um dos pés, o que poderia ajudar a explicar as interações entre a sensibilidade do pé e o controle postural de maneira mais precisa. Adicionalmente, o grupo de idosos não foi avaliado quanto à sarcopenia $\mathrm{e}$ neuropatias, que podem influenciar o controle postural ${ }^{21}$. Estudos futuros vão analisar a relação entre $\mathrm{CP}$ e sensibilidade, considerando, também, a descarga de peso em regiões específicas do pé, bem como as diferenças entre os membros inferiores e implicações sobre a sincronização intermembros por meio de avaliações do controle postural em cada um dos membros inferiores com medidas adicionais de cinemetria e baropodometria.

\section{CONCLUSÃO}

Os resultados sugerem que a amplitude ântero-posterior e a área do centro de pressão de idosos têm correlação com a sensibilidade plantar geral, mas não com regiões específicas do pé, enquanto que para jovens, a sensibilidade na região do antepé foi relacionada com o melhor controle postural. Sendo assim, é difícil determinar se uma região específica do pé possui maior relação com o controle postural em idosos.

\section{REFERÊNCIAS BIBLIOGRÁFICAS}

1. IBGE - Instituto Brasileiro de Geografia e Estatística. Estudos \& Pesquisas. Informação demográfica e socioeconômica. IBGE: Rio de Janeiro; 2010.

2. Patel M, Magnusson M, Kristinsdottir E, Fransson P. The Contribution of mechanoreceptive sensation on stability and adaptation in the young and elderly. Eur J Appl Physiol 2009;105:167-73. 
3. Horak F.B, Shupert C.L, Mirka A. Components of postural dyscontrol in elderly: A review. Neurobiol Aging 1989;10:727-38.

4. Kavounoudias A, Roll R, Roll JP. The plantar sole is a 'dynamometric map' for human balance control. Neuroreport 1998;9:3247-52.

5. Era P, Jokela J, Suominen H, Heikkinen E. Correlates of vibrotactile thresholds in men of different ages. Acta Neurol Scand 1986;74:210-7.

6. Kwan RL, Zheng Y-P, Cheing GL. The effect of aging on the biomechanical properties of plantar soft tissues. Clin Biomech 2010;25:601-5.

7. Lord S.R, Sherrington C, MENZ H.B. Falls in older people: Risk factors and strategies for prevention. Cambridge: Cambridge University Press 2001.

8. Patel M, Fransson P, Johansson R. Foam posturography: standing on foam is not equivalent to standing with decreased rapidly adapting mechanoreceptive sensation. Exp Brain Res 2010;208(4):519-27.

9. Stal F, Fransson P A, Magnusson M, Karlberg M. Effects of hypothermic anesthesia of the feet on vibration-induced body sway and adaptation. J Vestib Res 2003;13:39-52.

10. Wright WG, Ivanenko YP, Gurfinkel VS. Foot anatomy specialization for postural sensation and control. J Neurophysiol 2012;107(5):1513-21.

11. Mello R. G, Oliveira L. F, Nadal J. Anticipation mechanism in body sway control and effect of muscle fatigue. J Electromyogr Kinesiol 2007;17(6):739-46.

12. Mold J. W, Vesely S. K, Keyl B. A, Schenk J. B, Roberts M. The prevalence, predictors, and consequences of peripheral sensory neuropathy in older adults. J Am Board Fam Pract 2004;17(1):309-18.

13. Kwan RL, Zheng Y-P, Cheing GL. The effect of aging on the biomechanical properties od plantar soft tissues. Clin Biomech 2010;25:601-5.

14. Toledo D.R, Barela J.A. Sensory and motor differences between young and older adults: somatosensory contribution to postural control. Rev Bras Fisioter 2010;14(3):267-74.

15. Lord SR, Menz HB. Visual contributions to postural stability in older adults. Gerontology 2000;46(6):306-10.

16. Kars HJ, Hijmans JM, Geertzen JH, Zijlstra W. The effect of reduced somatosensation on standing balance: a systematic review. J Diabetes Sci Technol 2009;3(4):931-43.

17. Vandervoort AA, Chesworth BM, Cunninghan DA, Paterson DH, Rechnitzer PA, Koval JJ. Age and Sex Effects on mobility of human ankle. J Gerontol 1992;47:M17-M21.

18. Kavounoudias A, Roll R, Roll J P. Foot sole and ankle muscle inputs contribute jointly to human erect posture regulation. J Physiol 2001;532(3):869-878.

19. Bloem B R, Allum J H J, Carpenter M G, Honegger F. Is lower leg proprioception essential for triggering human automatic postural responses? Exp Brain Res 2000;130:375-91.

20. Dickstein R. Stance stability with unilateral and bilateral light touch of an external stationary object. Somatosensory \& Motor Research 2005;22(4):319-25.

21. Matsudo SM, Matsudo VKR, Barros Neto TL. Impacto do envelhecimento nas variáveis antropométricas, neuromotoras e metabólicas da aptidão física. Rev Bras Med Esporte 2000;8(4):21-32.
(1) 